\title{
Research of Enterprise Storage Ecosystem Based on Storage Theory and Nonlinear Discrete Optimization
}

\author{
Zixin Lu, Jiaqi Zhu, Yufeng Gui* \\ College of Science, Wuhan University of Technology, Wuhan, China \\ Email: *guiyufeng@whut.edu.cn
}

How to cite this paper: Lu, Z.X., Zhu, J.Q. and Gui, Y.F. (2018) Research of Enterprise Storage Ecosystem Based on Storage Theory and Nonlinear Discrete Optimization. Applied Mathematics, 9, 738-748. https://doi.org/10.4236/am.2018.96051

Received: June 1, 2018

Accepted: June 26, 2018

Published: June 29, 2018

Copyright (C) 2018 by authors and Scientific Research Publishing Inc. This work is licensed under the Creative Commons Attribution International License (CC BY 4.0).

http://creativecommons.org/licenses/by/4.0/

\section{(c) (i) Open Access}

\begin{abstract}
Warehousing and transferring strategies are an important part of business operations. The issue of optimal warehousing and transferring strategy is studied in this paper. Wal-Mart in Wuhan serves as an example to establish a $(s$, $S$ ) random storage strategy model, a Markov chain model, and a nonlinear discrete programming model, aiming at maximizing the profit per cycle of every branch and further maximizing the company's total profit per cycle. Among them, the random storage strategy model establishes a security zone of inventory for every branch, that is, it can meet consumers' demand without spending too much storage costs. The Markov chain model is used to get the probability of losing sales opportunities in every branch. The nonlinear discrete programming model takes into account the horizontal transferring among branches, which further maximizes the company's overall profit expectations. The three models above can be used to formulate inventory strategies, assess risks, and provide advice for every branch in order to form a complete storage ecosystem and provide constructive suggestions for the company's operations.
\end{abstract}

\section{Keywords}

Marketing Strategy, $(s, S)$ Random Storage Strategy, Markov Chain, Discrete Nonlinear Programming Model

\section{Introduction}

With the continuous progress of society, the scale of some companies is gradually expanding. The inventory management capability of an enterprise is one of the major manifestations of its profitability. The improper management of a 
company's inventory can cause its profits to decline. In severe cases, supply-sales chains can even break. For large-scale enterprises, the storage level of their branches and the coordination of inventory among their branches are a very important issue [1] [2] [3] [4].

At present, researchers have drawn some research results on the formulation of corporate goods inventory management strategies. For example, Singh [5] presented a conceptual methodology for inventory issue, forming a framework for companies to deal with inventory issues. Phouratsamay [6] studied the lot-sizing problem that occurs in a supply chain composed of a supplier and a retailer and found the problem is NP-hard when the inventory bounds are set on the supplier level. Archetti [7] integrated the inventory management problem with the pickup and delivery vehicle routing problems, showing the integrated policy cost much less than a non-integrated policy. Kong Shuxia [8] proposed the $(s, S)$ random storage strategy to study the store purchase plans. Zhu Laihui [9] also adopted the $(s, S)$ random storage strategy for the study of storage planning, but he studied special inventory like aviation ammunition.

The innovation of this paper lies in the combination of the $(s, S)$ random storage strategy model with the Markov chain and the nonlinear discrete programming model. It not only helps carry out the inventory plan of a single branch, but also studies the entire company's inventory ecosystem. This provides a more constructive proposal for corporate decision makers.

\section{Problem Analysis}

1) For every branch's storage plan, given that the demand for the product is random at each cycle, the assumption is that demand is subject to Poisson distribution. Storage costs and out-of-stock losses should be taken into account to achieve a balance of interests between the two. In addition, the lower limit and upper limit of inventory should be considered. Therefore, the $(s, S)$ random storage strategy is adopted to establish the storage strategy of every branch.

2) For the evaluation of the stock-out risk of every branch, in order to determine the stock-out probability of each shop, a Markov chain model is proposed. The inventory quantity at the beginning of each cycle of every branch is taken as the state variable. The distribution of demand and the state transfer matrix can also be list out according to the established storage strategy. Then the probability vector in steady state can be obtained. Finally, the full probability formula can be used to determine the probability of out-of-stock for every branch, that is, the probability of losing sales opportunities.

3) For the construction of the company's storage ecosystem, that is, the horizontal transfer plan among every branch, a discrete optimization model is established with the objective function of the total inventory cost and the out-of-stock loss cost after the entire store system is allowed to carry out horizontal transfer. The objective function is minimized by solving the optimal call-out and call-in amount of every branch. Finally, the best transfer plan can be obtained. 


\section{The Mathematical Model}

\subsection{The Single-Branch Storage Strategy}

Since the sales volume of the product in the store is random, the inventory of the product is checked at the end of each cycle so that the order plan for the next cycle can be formulated in time. In order to decrease the storage cost, the store's backlog of liquidity and the purchase at the end of the cycle should not be excessive, but it should not be too small, which will result in the shortage of goods in the next cycle and affect the turnover. In this case, the ordering strategy adopted in this paper is to formulate a lower bound $s$ and an upper bound $S$. When the stock at the end of the period is not less than $s$, the order will not be placed; when the stock is less than $s$, the order will be placed, and the order quantity will make the next week's inventory amount reach $S$. This strategy is called $(S, S)$ random storage strategy [8].

Poisson distribution was published by the French mathematician Siméon-Denis Poisson in 1838. It is suitable for describing the number of random events occurring within a unit of time. The Poisson distribution's probability density function is:

$$
p(X=k)=\frac{\lambda^{k}}{k !} \mathrm{e}^{-\lambda}, k=0,1, \cdots
$$

The parameter $\lambda$ of the Poisson distribution is the average incidence of random events per unit time (or unit area).

Take Wuhan Zongguan Xihui Branch as an example, it is known that the purchase cost per batch is 100 yuan, the profit of each product is 700 yuan, and the storage fee for each item is 100 yuan. If there is an out-of-stock situation, the loss of each item will be 700 yuan. Set the demand for the product during a period as $r$, which can be regarded as a random variable, and meet the Poisson distribution of the parameter $\lambda$. Because the estimated average demand for the item in each sales cycle in Wuhan Zongguan Xihui Branch is 4.1, so the value of $\lambda$ is set as 4.1.

Assuming that the end-of-cycle inventory is $x$, and the purchase volume is $u$, the inventory quantity at the beginning of the next cycle is $x+u$, and the storage amount in a sales cycle is $x+u-r$.

Assuming the expectation of a total profit for a sales cycle is $J$. Due to the fact that:

Total profit $=$ total sales of goods - incoming goods - storage fees.

So, when $u=0$,

$$
J=700 r-L(x)
$$

where $L(x)$ is the expectation of cost of storing the product and the cost of missing goods in a sales cycle, and its expression is:

$$
L(x)=100 \sum_{r=0}^{x}(x-r) p(r)+700 \sum_{r=x}^{\infty}(r-x) p(r)
$$


where $p(r)$ is the density function of Poisson distribution of the parameter 4.1.

When $u>0$,

$$
J=700 r-100-L(x+u)
$$

To sum up, the expression of one cycle of total profit is:

$$
J(u)=\left\{\begin{array}{l}
2770-L(x+u), u>0 \\
2870-L(x), u=0
\end{array}\right.
$$

The method is provided in this paper for solving the lower and upper bounds of the ordering strategy (again using Wuhan Zongguan Xihui Branch as an example):

1) Solving the upper bound $S$

When $u>0$, to maximize total profit, differentiate $J(u)$ :

$$
J(u+1)-J(u)=-100 \sum_{r=0}^{x+u} p(r)+700 \sum_{r=x+u+1}^{\infty} p(r)
$$

Due to $x+u=S$, then:

$$
J(u+1)-J(u)=-100 \sum_{r=0}^{S} p(r)+700 \sum_{r=S+1}^{\infty} p(r)
$$

Let it be 0 , then:

$$
\frac{\sum_{r=0}^{S} p(r)}{\sum_{r=S+1}^{\infty} p(r)}=7
$$

Due to $\sum_{r=0}^{\infty} p(r)=1$, so:

$$
\sum_{r=0}^{S} p(r)=0.875
$$

Since $r$ obeys the Poisson distribution with a parameter of 4.1 and $p(r)$ is its density function, the Poisson distribution quintile of the above formula yields $S$ $=6$.

2) Solving the lower bound $s$

If the branch purchases the commodity, the total profit expression is:

$$
J_{1}(x)=2770-L(S)
$$

where $S=6$ and $x$ is the current inventory quantity. If the branch chooses not to purchase the commodity, the total profit expression is:

$$
J_{2}(x)=2870-L(x)
$$

When $J_{1}(x) \geq J_{2}(x)$, in order to achieve the maximum total profit, the branch should promptly report the number of orders. From the calculation by Mathematica, the result below is achieved:

$$
L(x) \geq 464.167
$$


Because $L(5)=448.718, L(4)=661.271$, so when $x \leq 4$, the branch should purchase the commodity.

Therefore, for Wuhan Zongguan Xihui Branch, the following ordering strategy is established: At the end of each cycle, the inventories of commodity should be checked. When the inventories were less than or equal to 4 , the number of orders should be reported in time and the stocks should be increased to 6 .

Through the method above, the ordering strategy of the product in every branch within the $3^{\text {rd }}$ ring road in Wuhan can be carried out similarly. See Table 1 for details.

\subsection{Risk Assessment Model Based on Markov Chain}

Based on the strategy, in order to obtain the probability of losing sales opportunities at every branch, a stock-out probability calculation method based on the Markov chain [10] is used, as follows.

The inventory per cycle of the commodity is in a random state. It is only related to the amount of inventory in the previous cycle and there is no aftereffect in this state. That is, the inventory amount per cycle can be described by the Markov chain.

Take Wuhan Zongguan Xihui Branch as an example. The value of the inventory amount at the end of the commodity cycle may be $0,1,2,3,4,5,6$, and the initial value of inventory amount of each cycle may be 5,6 . Set $S_{n}$ to be the initial inventory of the $n^{\text {th }}$ cycle, then $S_{n} \in\{5,6\}$.

For the inventory condition of this product, the state transfer law is:

$$
S_{n+1}=\left\{\begin{array}{l}
S_{n}-r(n), r(n)<S_{n}-s \\
6, r(n) \geq S_{n}-s
\end{array}\right.
$$

where $r(n)$ is the demand for this product for the $n^{\text {th }}$ cycle, which obeys the Poisson distribution with a parameter of 4.1, which is:

$$
p(r(n)=k)=\frac{4.1^{k}}{k !} \mathrm{e}^{-4.1}
$$

Its distribution is shown in Table 2.

Table 1. The ordering strategy of every branch.

\begin{tabular}{cccc}
\hline Name of Branch & Parameter $\lambda$ & Lower Bound $s$ & Upper Bound $S$ \\
\hline 1) Wuhan Zongguan Xihui Branch & 4.1 & 4 & 6 \\
2) Nanhu City Plaza Branch & 3.2 & 3 & 7 \\
3) Wuhan Xudong Street Branch & 5.0 & 4 & 6 \\
4) Optics Valley City Branch & 4.0 & 4 & 6 \\
5) Wuhan Aoshan Branch & 3.7 & 4 & 5 \\
6) Hanyang Zhongjiacun Branch & 3.5 & 2 & 4 \\
7) Hanyang Branch & 2.3 & 2 & 3 \\
\hline
\end{tabular}


Table 2. Distribution of $r(n)$.

\begin{tabular}{ccccccccc}
\hline $\mathrm{r}(\mathrm{n})$ & 0 & 1 & 2 & 3 & 4 & 5 & 6 & $>6$ \\
\hline $\mathrm{P}$ & 0.0166 & 0.0679 & 0.1393 & 0.1904 & 0.1951 & 0.1600 & 0.1093 & 0.1214 \\
\hline
\end{tabular}

Then the state transition matrix for the inventory of this commodity is calculated as:

$$
p=\left[\begin{array}{ll}
p_{55} & p_{56} \\
p_{65} & p_{66}
\end{array}\right]=\left[\begin{array}{ll}
0.0166 & 0.9834 \\
0.0679 & 0.9321
\end{array}\right]
$$

The state transition probability is $a_{i}(n)=p\left(S_{n}=i\right), i=5,6$. Then:

$$
a(n)=\left[\begin{array}{l}
a_{5}(n) \\
a_{6}(n)
\end{array}\right]
$$

Due to $a(n+1)=a(n) \cdot p$, so:

$$
\left[\begin{array}{l}
a_{5}(n+1) \\
a_{6}(n+1)
\end{array}\right]=\left[\begin{array}{l}
a_{5}(n) \\
a_{6}(n)
\end{array}\right]\left[\begin{array}{ll}
0.0166 & 0.9834 \\
0.0679 & 0.9321
\end{array}\right]
$$

Because $\exists N, p^{N}>0$, so $p$ is a regular chain. There exists a unique limit state probability vector $w$. When $n \rightarrow+\infty$, the state probability $a(n) \rightarrow w . w$ is the steady-state probability which satisfies $w p=w$.

When $n \rightarrow+\infty$,

$$
p^{n}=\left[\begin{array}{ll}
0.0646 & 0.9354 \\
0.0646 & 0.9354
\end{array}\right]
$$

Then $w=[0.0646,0.9354]$. So when $n \rightarrow+\infty$, the state probability $a(n)=[0.0646,0.9354]$.

Let the probability of losing sales opportunities in the $n^{\text {th }}$ cycle be $p\left(r(n)>S_{n}\right)$, then:

$$
p\left(r(n)>S_{n}\right)=\sum_{i=5}^{6} p\left(r(n)>i \mid S_{n}=i\right) p\left(S_{n}=i\right)
$$

where $p\left(S_{n}=i\right)=w_{i}$. Then $p\left(r(n)>S_{n}\right)=12.85 \%$.

Therefore, the probability of losing sales opportunities in the ordering strategy of Wuhan Zongguan Xihui Branch is $12.85 \%$.

Through the method above, the probability of losing the sales opportunity in the ordering strategy of the product in every branch can be obtained respectively, as shown in Table 3.

\subsection{Construction of the Company's Storage Ecosystem}

In order to obtain the optimal transfer plan, a nonlinear discrete programming model is established.

Let $y_{i}$ be the stock amount of the $i^{\text {th }}$ branch at a certain time, $Q_{i}$ be the call-out amount or call-in amount of the $i^{\text {th }}$ branch at the time of transfer, and $Q_{i}$ should be taken as a positive value when called out, and it should be a negative value when called in. 
Table 3. Probability of losing sales opportunity.

\begin{tabular}{cc}
\hline Name of Branch & Probability of Losing Sales Opportunity \\
\hline 1) Wuhan Zongguan Xihui Branch & $12.85 \%$ \\
2) Nanhu City Plaza Branch & $11.9 \%$ \\
3) Wuhan Xudong Street Branch & $13.68 \%$ \\
4) Optics Valley City Branch & $11.78 \%$ \\
5) Wuhan Aoshan Branch & $8.94 \%$ \\
6) Hanyang Zhongjiacun Branch & $14.23 \%$ \\
7) Hanyang Branch & $10.75 \%$ \\
8) Lingjiao Lake Wanda Branch & $16.14 \%$ \\
\hline
\end{tabular}

When the transfer is completed, the amount of inventory in every branch becomes $y_{i}-Q_{i}$, and then the storage cost and the stock loss cost of every branch is:

$$
f\left(Q_{i}\right)=100 \sum_{r=0}^{y_{i}-Q_{i}}\left(y_{i}-Q_{i}-r\right) p(r)+700 \sum_{r=y_{i}-Q_{i}+1}^{\infty}\left(r-y_{i}+Q_{i}\right) p(r)
$$

Since the way of transferring goods is from stocked branches to out-of-stock branches, the quantity of mobilized goods is balanced, that is, there exists the constraint $\sum_{i=1}^{8} Q_{i}=0$. In addition, the objective function should also satisfy the limit of every branch's inventory. So the model is obtained.

$$
\begin{aligned}
& \min F(Q)=\sum_{i=1}^{n}\left\{100 \sum_{r=0}^{y_{i}-Q_{i}}\left(y_{i}-Q_{i}-r\right) p(r)+700 \sum_{r=y_{i}-Q_{i}+1}^{\infty}\left(r-y_{i}+Q_{i}\right) p(r)\right\} \\
& \text { s.t. }\left\{\begin{array}{l}
\sum_{i=1}^{n} Q_{i}=0 \\
0 \leq y_{i}-Q_{i} \leq S_{i}
\end{array}\right. \\
& j=1,2, \cdots, n
\end{aligned}
$$

This is a nonlinear programming problem with general constraints. Since the second derivative of $F(Q)$ exists and is greater than 0 , it is obvious that the objective function is a continuously differentiable convex function, and the constraint condition is linear. Therefore, this problem is also a convex programming which has an optimal set $\left\{Q_{i}^{*} \mid i=1,2, \cdots, 8\right\}$ which satisfies the constraints.

If the constraints are not considered, the optimal solution of the objective function $F(Q)$ can be obtained by differentiation: [11].

Let the first-order difference of $F(Q)$ to $Q_{i}$ be 0

$$
\Rightarrow P\left(\frac{y_{i}-\lambda_{i}-Q_{i}^{*}}{\lambda_{i}}\right)=1-\frac{100}{700+100} P(1)
$$

It can be seen from the above formula that the value of the optimal solution $Q_{i}$ that does not consider the constraints is related to $\lambda$, and $Q_{i}$ may take a positive value or a negative value. If the values of $Q_{i}$ are all greater than 0 , it 
means that there is no shortage of goods in every branch, then no branch needs to transfer the goods; otherwise, it means that every branch is out of stock and needs to replenish inventory from the manufacturer. Since the focus of this paper is the horizontal transfer strategy among branches, the premise is that the phenomenon of sufficient inventory and out-of-stock exists at the same time. Therefore, for the value of $Q_{i}$, the situation is not considered that the inventory of every branch is greater than or equal to 0 or all cases are less than or equal to 0 . It is assumed that these two conditions coexist.

In order to obtain the optimal value of $Q_{i}$, Lagrange multipliers $\alpha, \beta, \gamma$ are introduced: [11]

$$
l\left(Q_{i}, \alpha, \beta, \gamma\right)=F(Q)+\alpha^{*} \sum_{i=1}^{n} Q_{i}+\sum_{i=1}^{n} \gamma_{i}^{*}\left(Q_{i}-y_{i}\right)+\sum_{i=1}^{n} \beta_{i}^{*}\left(y_{i}-Q_{i}-S_{i}\right)
$$

Differentiate 1 :

First-order difference of $I$ to $Q_{i}$

$$
=700 \sum_{r=y_{i}-Q_{i}+1}^{\infty} p(r)-100 \sum_{r=0}^{y_{i}-Q_{i}} p(r)+\alpha^{*}+\gamma_{i}^{*}-\beta_{i}^{*}
$$

Then the equation set below is obtained:

$$
\left\{\begin{array}{l}
700 \sum_{r=y_{i}-Q_{i}+1}^{\infty} p(r)-100 \sum_{r=0}^{y_{i}-Q_{i}} p(r)+\alpha^{*}+\gamma_{i}^{*}-\beta_{i}^{*} \\
\gamma_{i}^{*}\left(Q_{i}-y_{i}\right)=0 \\
\beta_{i}^{*}\left(y_{i}-Q_{i}-S_{i}\right)=0 \\
\sum_{i=1}^{n} Q_{i}=0 \\
Q_{i}-y_{i} \leq 0 \\
y_{i}-Q_{i}-S_{i} \leq 0 \\
\lambda^{*}, \gamma_{i}^{*}, \beta_{i}^{*} \geq 0 \\
i=1,2, \cdots, 8
\end{array}\right.
$$

Obviously, the value of $\gamma_{i}^{*}$ and $\beta_{i}^{*}$ can only be 0 . Simplify the equation set above:

$$
\left\{\begin{array}{l}
P\left(\frac{y_{i}-\lambda_{i}-Q_{i}}{\lambda_{i}}\right)=1-\frac{100}{700+100} P(1)+\frac{\alpha^{*}}{700+100}, i=1,2, \cdots, 8 \\
\sum_{i=1}^{n} Q_{i}=0
\end{array}\right.
$$

Solve the set $\left\{Q_{i}^{*} \mid i=1,2, \cdots, 8\right\}$ through MATLAB. Round $Q_{i}^{*}$ with the constraints that $Q_{i}-y_{i} \leq 0 ; y_{i}-Q_{i}-S_{i} \leq 0$ so that the optimal value of $Q_{i}$ for every branch can be determined, as is shown in Table 4.

Substitute $Q_{i}^{*}$ into $F(Q)$, then $F\left(Q^{*}\right)=2018.27$.

Take three branches as an example, the transfer relationship among the branches is shown in Figure 1.

As is shown in Figure 1, for the case of the 3 branches, the transfer relations are divided into two layers: the first layer is the manufacturer supplying to the 


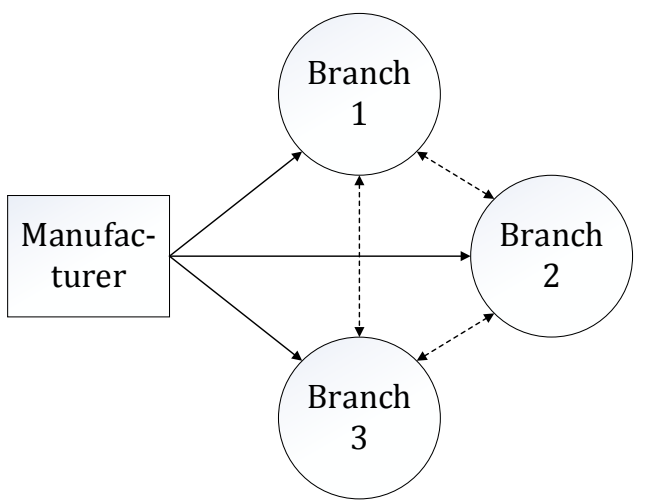

Figure 1. Transfer relations.

Table 4. Optimal value of $Q_{i}^{*}$ for every branch.

\begin{tabular}{ccccccccc}
\hline$i$ & 1 & 2 & 3 & 4 & 5 & 6 & 7 & 8 \\
\hline$Q_{i}^{*}$ & 2 & -1 & 1 & 0 & -3 & 1 & -3 & 3 \\
\hline
\end{tabular}

branches, and the second layer is the three branches transferring mutually according to the inventory situation.

Based on the principle of proximity, with the map as a reference, the desired transfer plan is obtained:

The excess stock of Wuhan Zongguan Xihui Branch and Hanyang Zhongjiacun Branch should be transferred to Hanyang Branch; the excess inventory of Wuhan Xudong Street Branch should be transferred to Nanhu City Plaza Branch; the excess inventory of Lingjiao Lake Wanda Branch should be transferred to Wuhan Aoshan Branch.

The expected number of shipments is 4 and the expected commodity transfer cost is 400 yuan. Therefore, in the case of considering the transfer of goods among branches, the total cost is 2418.27 yuan.

If the transfer of goods among branches is banned, the expected total cost is:

$$
\sum_{i=1}^{8}\left[100 \sum_{r=0}^{S_{i}}\left(S_{i}-r\right) p(r)+700 \sum_{r=S_{i}+1}^{\infty}\left(r-S_{i}\right) p(r)\right]=2696.92
$$

Therefore, the construction of transfer system helps increase the company's expectation of profits per cycle by 278.65 yuan.

\section{Model Assessment and Verification}

\subsection{Model Test of Single-Branch Storage Strategy}

The upper limit of every branch's inventory is 1 to 2 more than the estimated average demand. The lower bound of every branch inventory happens to be the rounding estimated average demand. Combining the result of risk assessment, every branch's purchasing strategy can suppress the probability of out-of-stock to be about $10 \%$, and the probability of losing sales opportunities is small. Therefore, the storage strategy formulated in this paper is realistic and effective. 


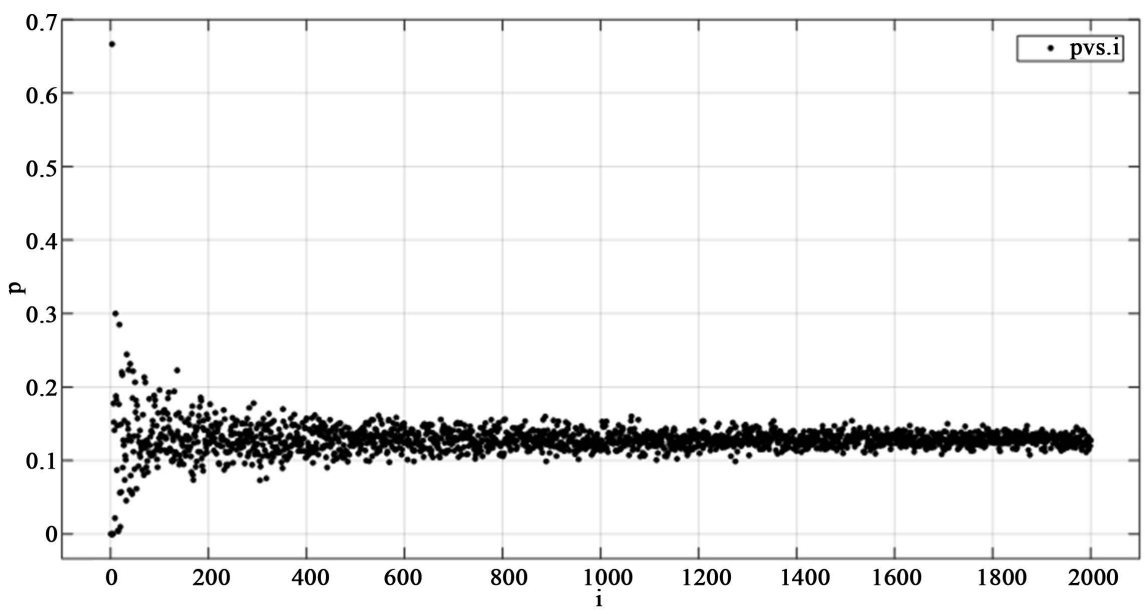

Figure 2. Simulation of stock-out probability for Wuhan Zongguan Xihui Branch.

\subsection{Model Test of Risk Assessment Model}

For every branch's stock-out probability, MATLAB is adopted to carry out the simulation. The simulation result shows that the stock-out probability is converging. Take Wuhan Zongguan Xihui Branch as an example, the stock-out probability of Wuhan Zongguan Xihui Branch is $12.85 \%$. MATLAB is adopted to generate a sample from Poisson distribution with sample size $n$, and calculate the stock-out probability for $n$ in the range of 1 to 2000. The stock-out probability figure is shown in Figure 2.

From the figure above, when $n$ is large enough, the stock-out probability converges to about $12.85 \%$. Therefore, the stock-out probability obtained from the Markov chain model is reasonable.

\section{Conclusions and Suggestions}

For Wal-Mart's products of this kind, the expected number of transfer among the branches per cycle is 4 times, and the expectation of profit increase is 278.65 yuan, that is, profits increase by about $10 \%$. The increase in profit is in line with the facts and the number of transfers is within a reasonable range. Therefore, the model is reasonable and feasible, and the model results have practical significance.

A new model for the storage problem of enterprises is proposed in this paper. It comprehensively uses $(s, S)$ random storage strategy, Markov chain and nonlinear discrete programming model to develop a complete storage system for the enterprise. From the model results above, it can be known that the whole system designed for the company performs well. The storage system has a good performance of extrapolation and is worthy of promotion. It can bring considerable benefits to more companies.

\section{References}

[1] Suo, M.Q. (2013) Study on Regional Resource Optimization Method Based on Sto- 
rage Theory. North China Electric Power University, Beijing.

[2] Luo, W.T. (2012) Mathematical Model of Product Sales Storage Problem. China Business, 6, 102-103.

[3] Gao, Y.F. (2008) The (s, S) Probability Model for Random Storage Problems. Market Modernization, 33, 35.

[4] Zhang, L. (2010) Research on Product Storage Optimization and System Implementation. Jilin University, Changchun.

[5] Singh, D. and Verma, A. (2018) Inventory Management in Supply Chain. Materials Today: Proceedings, 5, 3867-3872. https://doi.org/10.1016/j.matpr.2017.11.641

[6] Phoutatsamay, S.-L., Kedad-Sidhoum, S. and Pascual, F. (2018) Two-Level Lot-Sizing with Inventory Bounds. Discrete Optimization. https://doi.org/10.1016/j.disopt.2018.05.001

[7] Archetti, C., Christiansen, M. and Grazia Speranza, M. (2018) Inventory Routing with Pickups and Deliveries. European Journal of Operational Research, 268, 314-324. https://doi.org/10.1016/j.ejor.2018.01.010

[8] Kong, S.X. and Han, Z.Y. (2007) A Random Storage Strategy for Ordering Problems. Market Modernization, 32, 34.

[9] Zhu, L.H., Cao, W. and Zhang, Y. (2012) Aviation Ammunition Random Storage Strategy Model. Journal of Sichuan Armament, 33, 24-25.

[10] Xu, Y. (2014) Research on Network Discrete System Filtering and Control Based on Markov Chain. Zhejiang University, Hangzhou.

[11] Yang, J., Tang, Y.L. and Lin, B. (2006) Horizontal Replenishment Optimization Model of Distribution System under Random Demand. China Storage and Transportation, 5, 98-99. 